\title{
Advanced photoacoustic image reconstruction using the k-Wave toolbox
}

\author{
B. E. Treeby*, J. Jaros ${ }^{\dagger}$, and B. T. Cox* \\ *Department of Medical Physics and Biomedical Engineering, University College London, UK \\ ${ }^{\dagger}$ Faculty of Information Technology, Brno University of Technology, Czech Republic
}

\begin{abstract}
Reconstructing images from measured time domain signals is an essential step in tomography-mode photoacoustic imaging. However, in practice, there are many complicating factors that make it difficult to obtain high-resolution images. These include incomplete or undersampled data, filtering effects, acoustic and optical attenuation, and uncertainties in the material parameters. Here, the processing and image reconstruction steps routinely used by the Photoacoustic Imaging Group at University College London are discussed. These include correction for acoustic and optical attenuation, spatial resampling, material parameter selection, image reconstruction, and log compression. The effect of each of these steps is demonstrated using a representative in vivo dataset. All of the algorithms discussed form part of the open-source $\mathrm{k}$-Wave toolbox (available from http://www.k-wave.org).
\end{abstract}

\section{INTRODUCTION}

Forming an image from measured time domain signals is an essential step in photoacoustic tomography (PAT). Superficially, this would appear to be a solved problem, particularly given the large number of published photoacoustic images. ${ }^{1}$ Indeed, commercial photoacoustic scanners are now available that can generate images in real time, ${ }^{2}$ and exact reconstruction formulae for canonical geometries have existed in the mathematical literature for some time. ${ }^{3}$ However, in the practical case, there are many complicating factors that make it difficult to obtain high-resolution images with good signal-to-noise. These include the data being incomplete (e.g., because the detection aperture is limited or spatially undersampled) ${ }^{4}$ filtering effects (e.g., because the transducer elements have limited sensitivity and bandwidth, and have a finite size), ${ }^{5,6}$ limited penetration depth (due to optical and acoustic attenuation), ${ }^{1,7}$ and uncertainties in the material properties needed for the reconstruction. ${ }^{8,9}$ While advances have been made to address many of these challenges, the rapid growth in the development and application of photoacoustic technology means that there is often a disconnect between researchers developing new algorithms and those performing in vivo imaging studies. In this paper, the preprocessing, image reconstruction, and post-processing steps routinely used by the Photoacoustic Imaging Group at University College London (UCL) to generate high-resolution photoacoustic images are discussed. The purpose is to provide insight into the impact of applying different techniques on reconstructed photoacoustic images. All of the algorithms discussed form part of the open-source $\mathrm{k}$-Wave image reconstruction toolbox developed at UCL (available from http://www.k-wave.org) ${ }^{10}$ This makes it easy for other researchers to apply them to their own datasets.

\section{DATASET AND ACQUISITION PARAMETERS}

The dataset used to demonstrate the different image reconstruction steps is taken from Ref. 11 (see Fig. 3(f) and front cover). This is an in vivo dataset of the blood vasculature and a xenograft (or tumour) composed of K562 cells labelled with a tyrosinase-based genetic reporter taken in the flank of a nude mouse. The dataset was acquired using a photoacoustic scanning system based on a planar Fabry-Perot interferometer. ${ }^{12}$ This was used as a 2 D detection array with $142 \times 141$ detection elements (giving a total of 20,022 time domain waveforms), an element separation of $100 \mu \mathrm{m}$ (giving a scan area of $14.2 \times 14.1 \mathrm{~mm}$ ), and an optically defined element size of $22 \mu \mathrm{m} .{ }^{11}$ The $-3 \mathrm{~dB}$ bandwidth of the detection system was $0.35-22 \mathrm{MHz}$, and the three-sigma noise equivalent

Send correspondence to b.treeby@ucl.ac.uk.

Photons Plus Ultrasound: Imaging and Sensing 2016, edited by Alexander A. Oraevsky, Lihong V. Wang Proc. of SPIE Vol. 9708, 97082P · (C) 2016 SPIE · CCC code: 1605-7422/16/\$18 - doi: 10.1117/12.2209254 

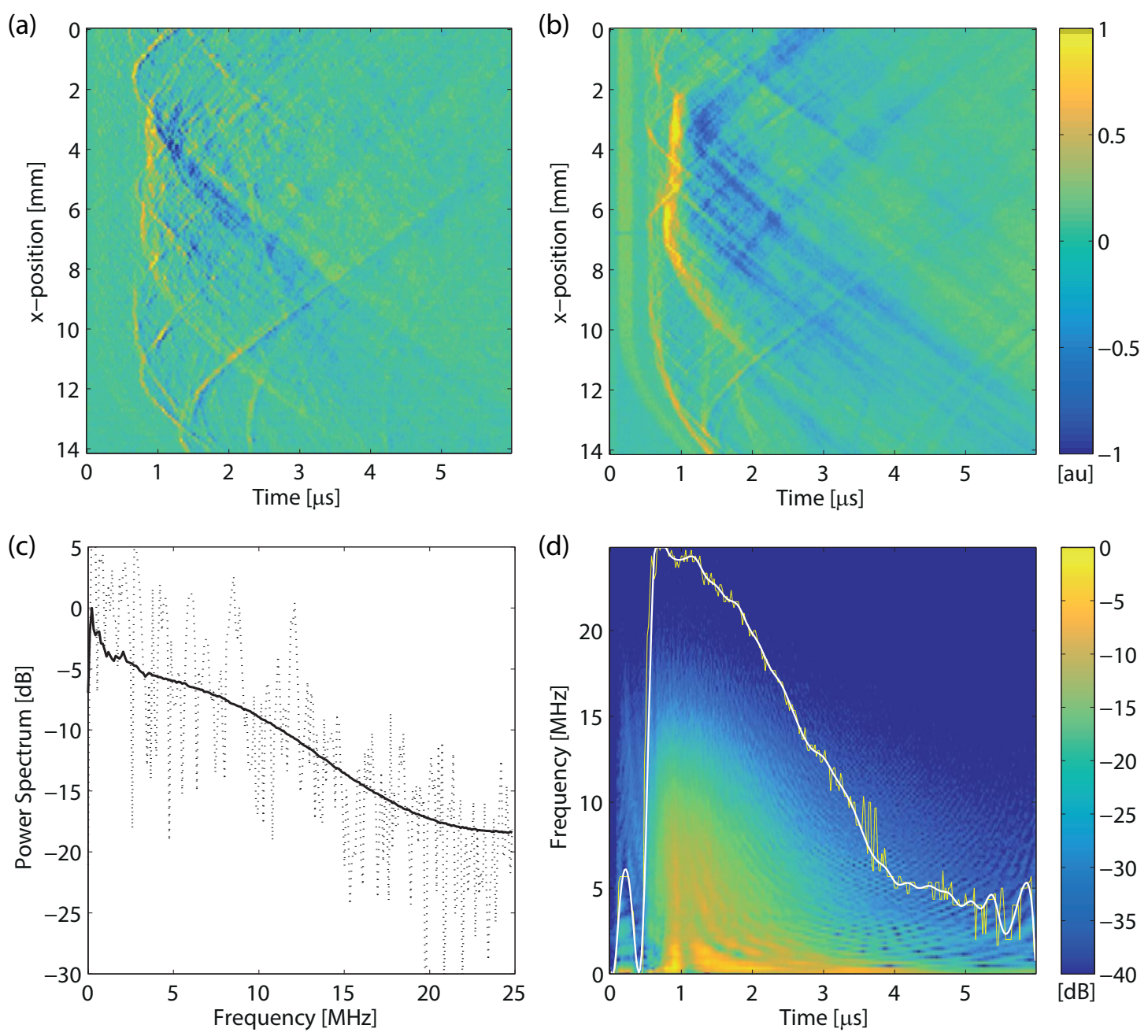

Figure 1. (a) Recorded time series data as a function of $x$-position and time at a $y$-position of $9 \mathrm{~mm}$. (b) Summation of the recorded time series data across all $y$-positions. (c) Power spectrum of the recorded time domain photoacoustic signals. The solid line shows the spectrum averaged across all signals, and the dashed line shows the spectrum of the signal recorded in the centre of the array. (d) Average time frequency distribution of the recorded signals. The yellow (thin) and white (thick) lines show the filter cutoff frequency before and after spline fitting, respectively.

pressure over a $20 \mathrm{MHz}$ bandwidth was $200 \mathrm{~Pa}$. Time domain signals at each detector position were acquired sequentially at a sampling frequency of $50 \mathrm{MHz}$ with no averaging, and the entire dataset took 6 minutes and 40 seconds to obtain using a $50 \mathrm{~Hz}$ excitation laser. Signal acquisition was triggered using a photodiode, such that the beginning of each time trace was synchronised with the excitation laser pulse reaching the tissue surface.

One line scan (as a function of $x$-position and time) from the recorded time series data is shown in Fig. 1(a). Bipolar signals from different optically absorbing tissue structures are clearly visible, which illustrates the low-noise and wide-bandwidth of the measurement system. ${ }^{12} \mathrm{~A}$ summation of the recorded time series data across the $y$ scanning dimension is shown in Fig. 1(b). The strongest signals appear around $1 \mu \mathrm{s}(\sim 1.5 \mathrm{~mm})$, however, features are still visible after at least $3 \mu \mathrm{s}(\sim 4.5 \mathrm{~mm})$. The power spectrum of the recorded data is shown in Fig. 1(c). The black line shows the spectrum averaged across all the signals, and the dotted line shows the spectrum of the signal recorded in the centre of the array. This demonstrates the broadband nature of the acquired photoacoustic signals, with energy from $350 \mathrm{kHz}$ (the low frequency cutoff of the acquisition system) up to approximately $20 \mathrm{MHz}$. 


\section{IMAGE RECONSTRUCTION AND PROCESSING STEPS}

\subsection{Workflow}

The pre-processing, reconstruction, and post-processing steps used to reconstruct the dataset shown in Fig. 1 are outlined below. The same procedure is routinely followed for most recent in vivo imaging studies using the Fabry-Perot scanner published by the Photoacoustic Imaging Group at UCL, e.g., Refs. 13-15.

1. Correct for acoustic attenuation in the time series data

2. Select sound speed that maximises the sharpness of the reconstructed image

3. Spatially upsample the acquired data to improve image resolution

4. Reconstruct the photoacoustic image

5. Correction for optical attenuation in the image data

6. Apply image processing and segmentation techniques as appropriate

7. Display as maximum intensity projection (MIP)

These steps are discussed in the following sections, with further details given in the references. The MATLAB and $\mathrm{k}$-Wave functions used to perform these steps are also described. Note, to illustrate the effect of the individual steps on the final reconstructed image, the images displayed in each section are reconstructed cognisant of details discussed in other sections. In particular, the optimum sound speed is always used, except where otherwise noted.

\subsection{Acoustic attenuation compensation}

It is well known that soft biological tissue is acoustically absorbing, with the experimentally observed attenuation following a frequency power law of the form $\alpha=\alpha_{0} f^{y}$. Due to the broadband nature of the ultrasound waves generated in photoacoustics, this causes a depth-dependent magnitude error and blurring of features in the reconstructed image. Applying compensation for acoustic attenuation can correct for these errors and improve the visibility and resolution of deeper vessels. ${ }^{16,17}$ Here, attenuation compensation is performed using time-variant filtering, which applies the correction directly to the time series data before reconstruction. ${ }^{18}$ This approach is very flexible, and can be applied regardless of the acquisition system, geometry, or the reconstruction method used. The algorithm works by applying a non-stationary convolution matrix to each recorded time series $p_{\text {att }}$, where

$$
\left[\begin{array}{c}
p_{\text {corr }}\left(t_{1}\right) \\
\vdots \\
p_{\text {corr }}\left(t_{N}\right)
\end{array}\right]=\left[\begin{array}{ccc}
F\left(t_{1}, \tau_{1}\right) & \ldots & F\left(t_{1}, \tau_{N}\right) \\
\vdots & & \vdots \\
F\left(t_{N}, \tau_{1}\right) & \ldots & F\left(t_{N}, \tau_{N}\right)
\end{array}\right]\left[\begin{array}{c}
p_{\text {att }}\left(\tau_{1}\right) \\
\vdots \\
p_{\text {att }}\left(\tau_{N}\right)
\end{array}\right] .
$$

The matrix $F$ is constructed to allow attenuation compensation as a function of both frequency and travel distance (or time).$^{18}$ In $\mathrm{k}$-Wave, this is applied using the function attenComp

$$
\text { sensor_data }=\text { attenComp (sensor_data, dt, c0, a0, y); }
$$

where sensor_data is a $2 \mathrm{D}$ matrix containing the recorded time series $p_{\text {att }}$ in each row, $\mathrm{dt}$ is the size of the time step in units of $\mathrm{s}, \mathrm{c} 0$ is the sound speed in units of $\mathrm{m} / \mathrm{s}, \mathrm{a} 0$ is the power law absorption prefactor in units of $\mathrm{dB} /\left(\mathrm{MHz}^{y} \mathrm{~cm}\right)$, and $\mathrm{y}$ is the power law absorption exponent. This function also automatically selects a cutoff frequency for the attenuation compensation (to stop high frequency noise being amplified) based on the average time-frequency distribution of the signals. ${ }^{18}$

To compensate for acoustic attenuation in the dataset shown in Fig. 1, the power law absorption parameters were set to those of breast tissue, with $\mathrm{a} 0=0.75$ and $\mathrm{y}=1.5{ }^{19}$ As the acoustic absorption parameters in 

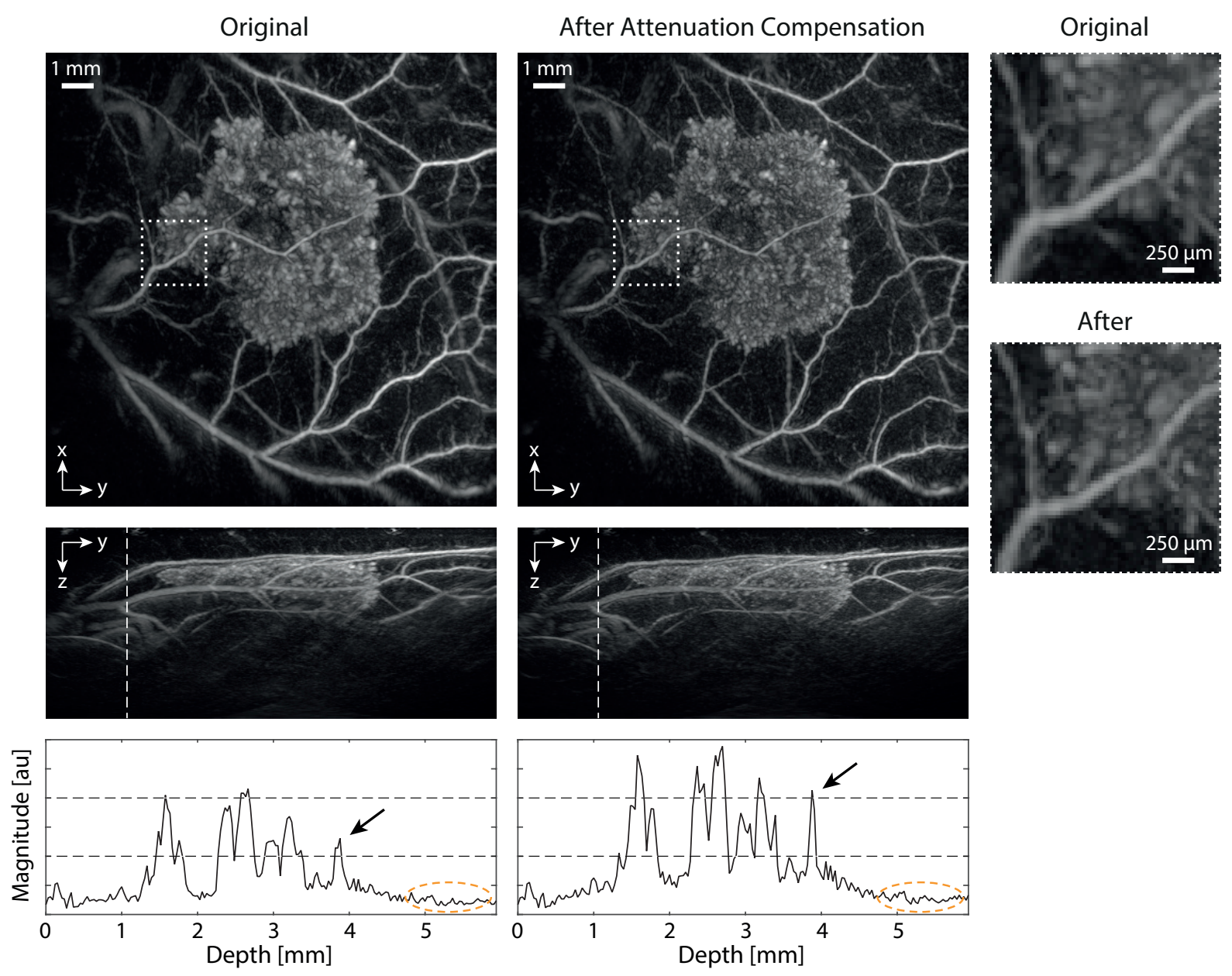

Figure 2. Three-dimensional reconstructions of the dataset shown in Fig. 1 with and without attenuation compensation. The three vertical panels show maximum intensity projections (MIPs) through the depth and lateral directions, and a one-dimensional profile through the lateral MIP at the location shown with a dashed white line. Magnification of the depth MIPs within the square dotted boxes is shown in the two right panels. Including attenuation compensation increases the sharpness of the vasculature and tumour, and improves the visibility and resolution of deeper vessels (arrows) without increasing the noise floor (circled region).

murine tissue are not well characterised, the average value in breast tissue is an apposite choice for soft-tissue containing a range of tissue types. The noise threshold and energy threshold used to select the filter cutoff frequency were set to $5 \%$ and $95 \%$, respectively. The average time frequency distribution and the automatically selected filter cutoff frequency are shown in Fig. 1(d). Reconstructed images with and without acoustic attenuation compensation are shown in Fig. 2 for comparison. The tumour can be clearly seen as the sponge like structure in the centre of the image, along with the surrounding blood vasculature. When attenuation compensation is included, the sharpness of the vasculature and tumour is increased. This is particularly noticeable in the magnified images, which show an improvement down to the voxel level. The visibility and resolution of deeper vessels is also improved, as shown in the one-dimensional profiles. For example, the visibility and sharpness of the vessel denoted with the black arrows has significantly increased, without a corresponding increase in the noise floor (circled region). It is useful to point out that these improvements are not an image processing trick; they arise directly from rectifying the acoustic losses that physically occur as the photoacoustic waves propagate through tissue. 
Regarding computational time, acoustic attenuation compensation using time variant filtering is very fast to apply. Using a desktop PC with an 8-core Intel Xeon E5-1660 v3 @ $3 \mathrm{GHz}$ processor running MATLAB $2015 \mathrm{a}$, the attenComp function took $3.1 \mathrm{~s}$ to calculate the average time frequency distribution, $0.45 \mathrm{~s}$ to select the cutoff frequency for the filter, $0.042 \mathrm{~s}$ to create the filter, and $0.19 \mathrm{~s}$ to apply the correction to all 20,022 time domain waveforms. If the filter cutoff frequency is chosen manually (based on the noise floor in the power spectrum for example ${ }^{20}$ ), there is no need to calculate the time frequency distribution, and the correction is even faster to apply.

\subsection{Sound speed selection}

The reconstruction of photoacoustic images requires knowledge of the sound speed within the medium so timeof-flight measurements can be correctly mapped back to the initial pressure distribution. Most reconstruction algorithms routinely used assume a constant value of sound speed. However, for in vivo imaging, the true value of sound speed is usually unknown. It is possible to estimate an appropriate value by systematically modifying the sound speed until the sharpness of the reconstructed image is maximised. ${ }^{8,21}$ This is based on the premise that features in the imaging volume are inherently sharp, and thus the correct sound speed is the one that produces the sharpest looking image. In k-Wave, sharpness is evaluated using the function sharpness. ${ }^{21} \mathrm{By}$ default, this uses a sharpness metric or focus function based on a simple finite difference gradient calculation known as the Brenner gradient. In $2 \mathrm{D}$ this is given by

$$
\mathrm{F}_{\text {brenner }}=\sum_{x, y}\left(f_{x+2, y}-f_{x, y}\right)^{2}+\left(f_{x, y+2}-f_{x, y}\right)^{2} .
$$

The sound speed value that maximises the sharpness metric can then be found by looping through a range of values as shown below, or using simple optimisation routines (e.g., fminbnd in MATLAB).

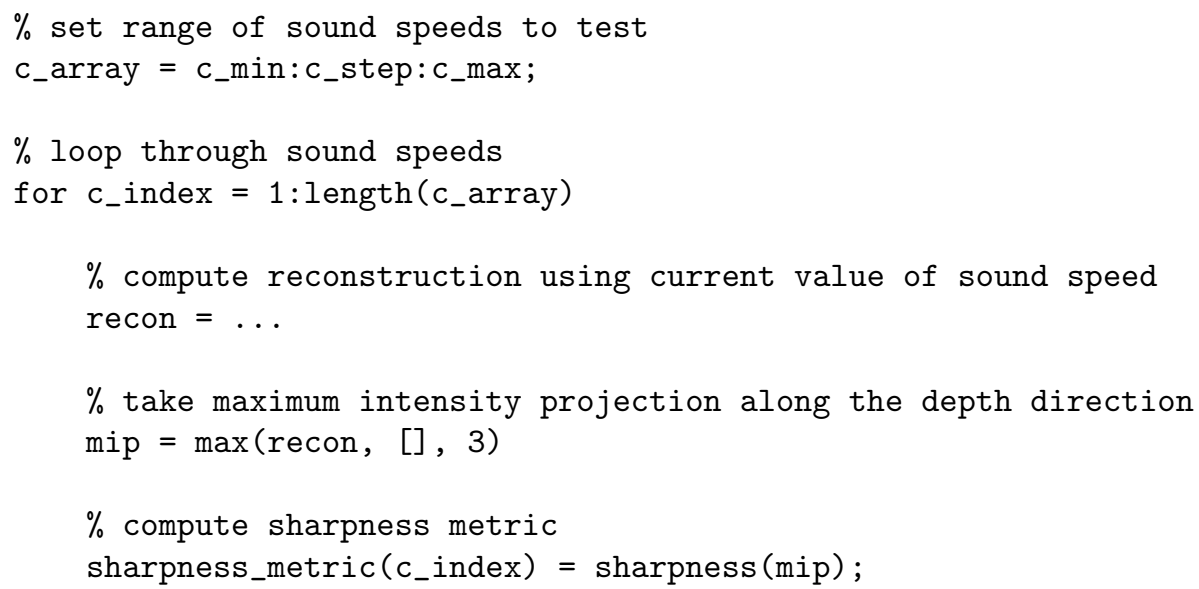

Figure 3(a) shows depth direction (enface) maximum intensity projections (MIPs) of the reconstructed tumour image using six different values of sound speed from $1400 \mathrm{~m} / \mathrm{s}$ to $1600 \mathrm{~m} / \mathrm{s}$. As the sound speed is increased, the image is gradually focused and then defocused again. The corresponding focus function calculated using Eq. (2) is shown in Fig. 3(b). In this case, the focus function is unimodal, with a peak at $1515 \mathrm{~m} / \mathrm{s}$ (shown with the dashed line). This is within the range of physiological values between fat (1430 
(a)
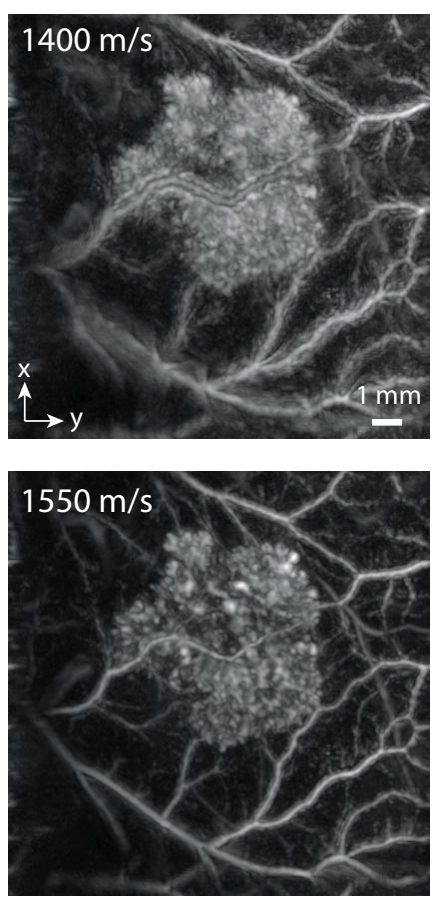
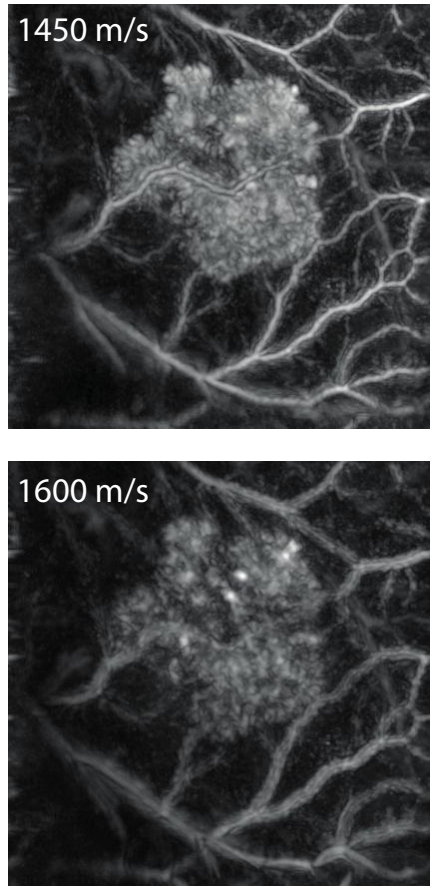
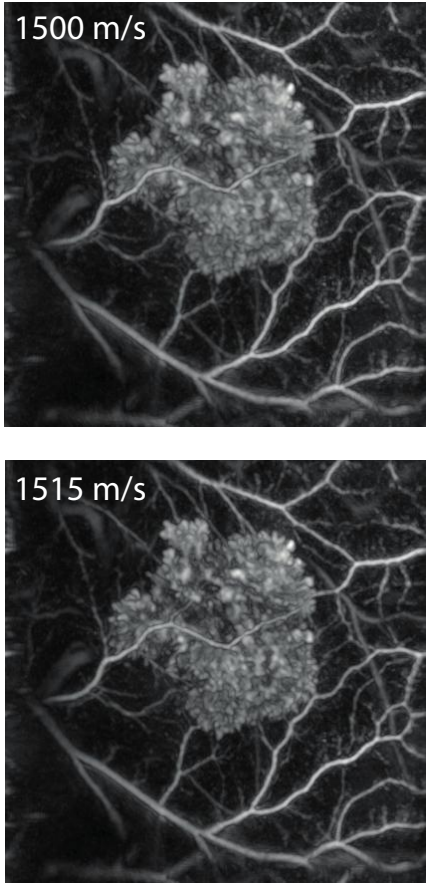

(b)

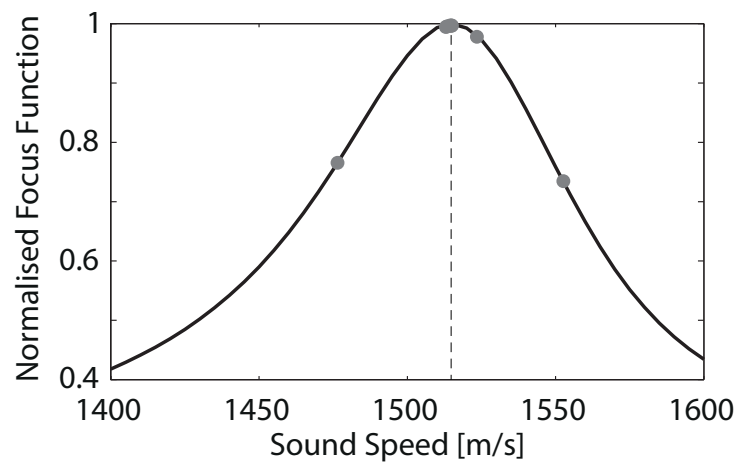

Figure 3. (a) Depth direction maximum intensity projections of the reconstructed image using different values for sound speed. A focusing and then defocusing can be noticed as the sound speed is increased. The reconstructed image using the sound speed that maximises the focus function (sharpness metric) is shown in the bottom right panel. (b) Variation of the focus function for sound speed values between 1400 and $1600 \mathrm{~m} / \mathrm{s}$. The focus function is unimodal, and the value of sound speed that maximises the focus function is shown with a dashed line. The steps used by fminbnd in MATLAB to find the maximum are shown with the grey dots.

$\mathrm{m} / \mathrm{s})$ and muscle $(1580 \mathrm{~m} / \mathrm{s}) .{ }^{19}$ The reconstructed image using the optimised value for sound speed is shown in the bottom right panel of Fig. 3(a). The computational cost of computing the MIP and sharpness metric are negligible, thus the main cost of this approach is the repeated image reconstruction that must be performed. Using fminbnd in MATLAB, the optimum value for sound speed is found in 6 steps, shown as the grey dots in Fig. 3(b). Combined with an optimised C++ version of the FFT-based algorithm described in Sec. 3.5, the complete autofocusing procedure takes less than 5 seconds.

\subsection{Upsampling}

Due to practical constraints of using the Fabry-Perot scanning system (including animal scanning times and acquisition memory depth), the temporal sampling used for in vivo imaging studies is typically higher than 
the spatial sampling (the same is true of most photoacoustic and ultrasound scanners). For the sampling parameters used for the tumour dataset, the maximum supported frequencies due to the temporal and spatial sampling are

$$
f_{\max , t}=\frac{1}{2 \Delta t}=25 \mathrm{MHz} \quad>\quad f_{\max , x}=\frac{c}{2 \Delta x}=7.575 \mathrm{MHz} .
$$

As shown in Fig. 1(c), the acquired photoacoustic signals are very broadband, containing energy up to 20 MHz. This means the acquisition is spatially undersampled. Thus, if the image is reconstructed onto a grid defined by the spatial acquisition parameters, higher frequency information contained in the temporal signals will not be used, reducing resolution. To overcome this, the grid parameters used for the reconstruction can be spatially upsampled. This is demonstrated in Fig. 4, where the tumour image has been reconstructed using time reversal with upsampling factors of 1 (no upsampling), 2 and 3 . This corresponds to a grid spacing (and maximum supported frequency) of $100 \mu \mathrm{m}(7.575 \mathrm{MHz}), 50 \mu \mathrm{m}(15.15 \mathrm{MHz})$, and $33 \mu \mathrm{m}(22.725 \mathrm{MHz})$, respectively. To allow a fair comparison, all three reconstructed images have been resampled to the same resolution for display using Fourier interpolation (interpftn in k-Wave). There is a very clear improvement with an upsampling factor of 2 compared to no upsampling. The small vessels are more visible, and there is much greater detail in the tumour mass. In comparison, there is little perceptible different between the reconstructed images with 2 and 3 times upsampling, despite the latter allowing almost the full range of frequencies contained in the temporal signals to be used in the reconstruction.

To examine this in more detail, the tumour dataset was reconstructed with an upsampling factor of 3 after first low-pass filtering the time signals. The reconstructed images for filter cutoff frequencies between $12 \mathrm{MHz}$ and $4 \mathrm{MHz}$ are shown in Fig. 5. For filter cutoff frequencies above $12 \mathrm{MHz}$, there was no discernible change in the image. At $12 \mathrm{MHz}$, the magnitude of the reconstructed image starts to decrease, and there is a slight reduction in high frequency variations at the voxel level. At $10 \mathrm{MHz}$, the main features are all still discernible, but begin to become noticeably softer. This trend continues down to $4 \mathrm{MHz}$, where the tumour and main vessels are still visible, but significantly blurred. Thus for this dataset, qualitatively it would appear that the frequency content up to $\sim 12 \mathrm{MHz}$ has a perceptible impact on the reconstructed image. This explains why there is no noticeable difference seen between upsampling factors of 2 and 3 shown in Fig. 4.

The computational cost of using upsampling (particularly with time reversal image reconstruction) is that the image reconstruction must be performed using a larger computational grid. The grid size, compute time, and memory usage for the three reconstructions shown in Fig. 4 (using the 20,022 recorded time series) are given in Table 1. The reconstructions were performed using an optimised $\mathrm{C}++$ /CUDA version of $\mathrm{k}$-Wave running on an NVIDIA GeForce GTX TITAN X graphics processing unit (GPU). ${ }^{22}$ Even at the largest scale, the reconstruction takes less than 30 seconds and uses less than 4 GB of memory.

Table 1. Summary of grid size and compute time to reconstruct the tumour image using time reversal with different upsampling factors.

\begin{tabular}{llll}
\hline Upsampling Factor & Grid Size & Compute Time & Memory Usage \\
\hline 1 & $162 \times 162 \times 96$ & $1.5 \mathrm{~s}$ & $419 \mathrm{MB}$ \\
2 & $324 \times 324 \times 144$ & $8.1 \mathrm{~s}$ & $1320 \mathrm{MB}$ \\
3 & $450 \times 450 \times 216$ & $25.3 \mathrm{~s}$ & $3368 \mathrm{MB}$ \\
\hline
\end{tabular}

\subsection{Reconstruction methods}

Two algorithms are routinely used for reconstructing the datasets acquired using the planar Fabry-Perot scanning system. The first is a fast one-step method based on an interpolation between spatial and temporal frequency performed in the Fourier domain as shown below (kspacePlaneRecon in k-Wave). ${ }^{23,24}$

$$
p(x, y, t) \stackrel{\mathrm{FFT}}{\longrightarrow} P\left(k_{x}, k_{y}, \omega\right) \stackrel{\frac{\omega}{c^{2}}=k_{x}^{2}+k_{y}^{2}+k_{z}^{2}}{\longrightarrow} H\left(k_{x}, k_{y}, k_{z}\right) \stackrel{\mathrm{IFFT}}{\longrightarrow} h(x, y, z)
$$

The second is time reversal, where the detected signals are propagated back into the domain in time reversed order using a numerical model of the acoustic forward problem (kspaceFirstOrder3D in k-Wave). ${ }^{17,25,26}$ 

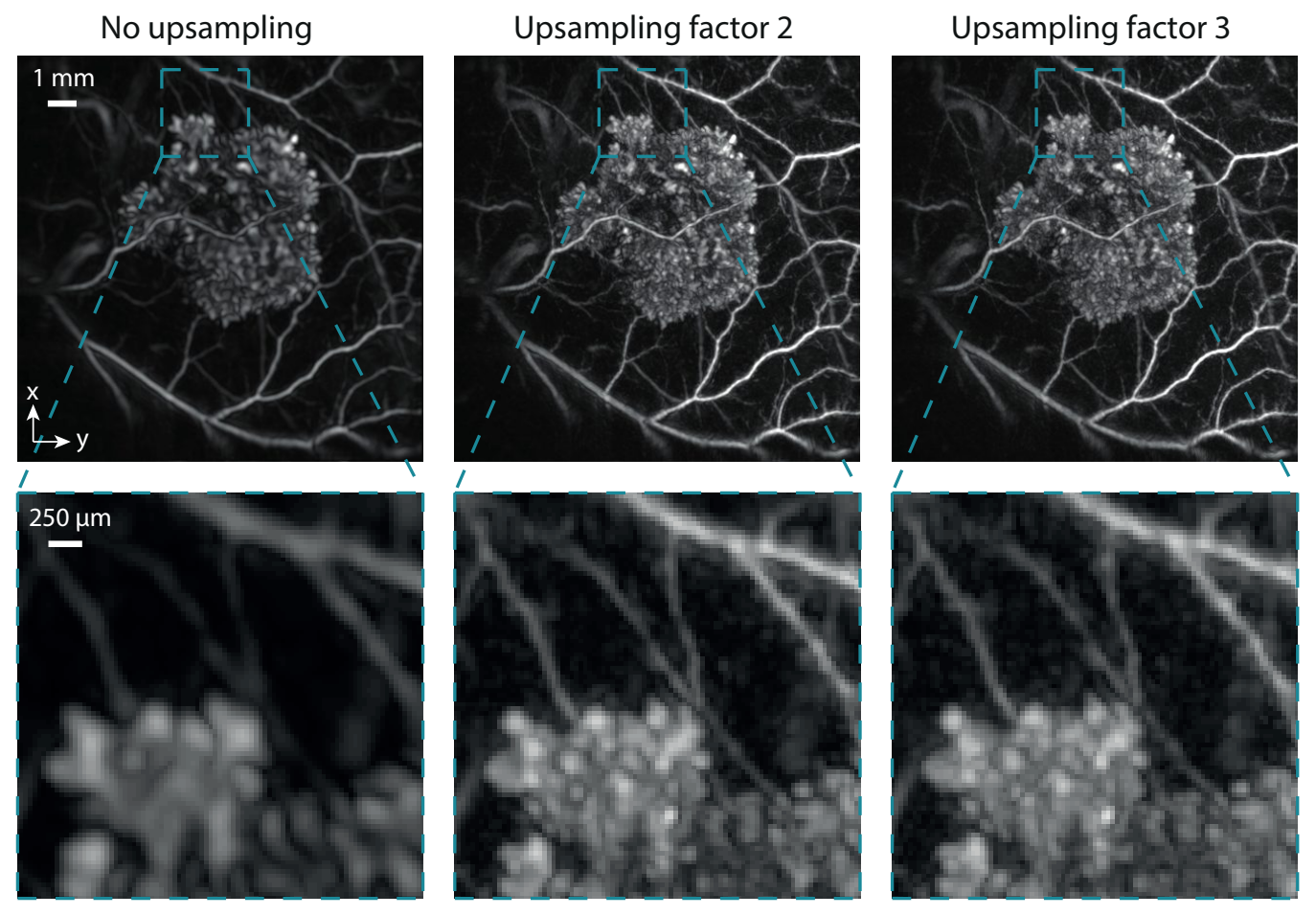

Figure 4. Effect of spatial upsampling on the resolution of reconstructed photoacoustic images. The maximum supported frequency for the three reconstructions is $7.575 \mathrm{MHz}, 15.15 \mathrm{MHz}$, and $22.725 \mathrm{MHz}$, respectively.
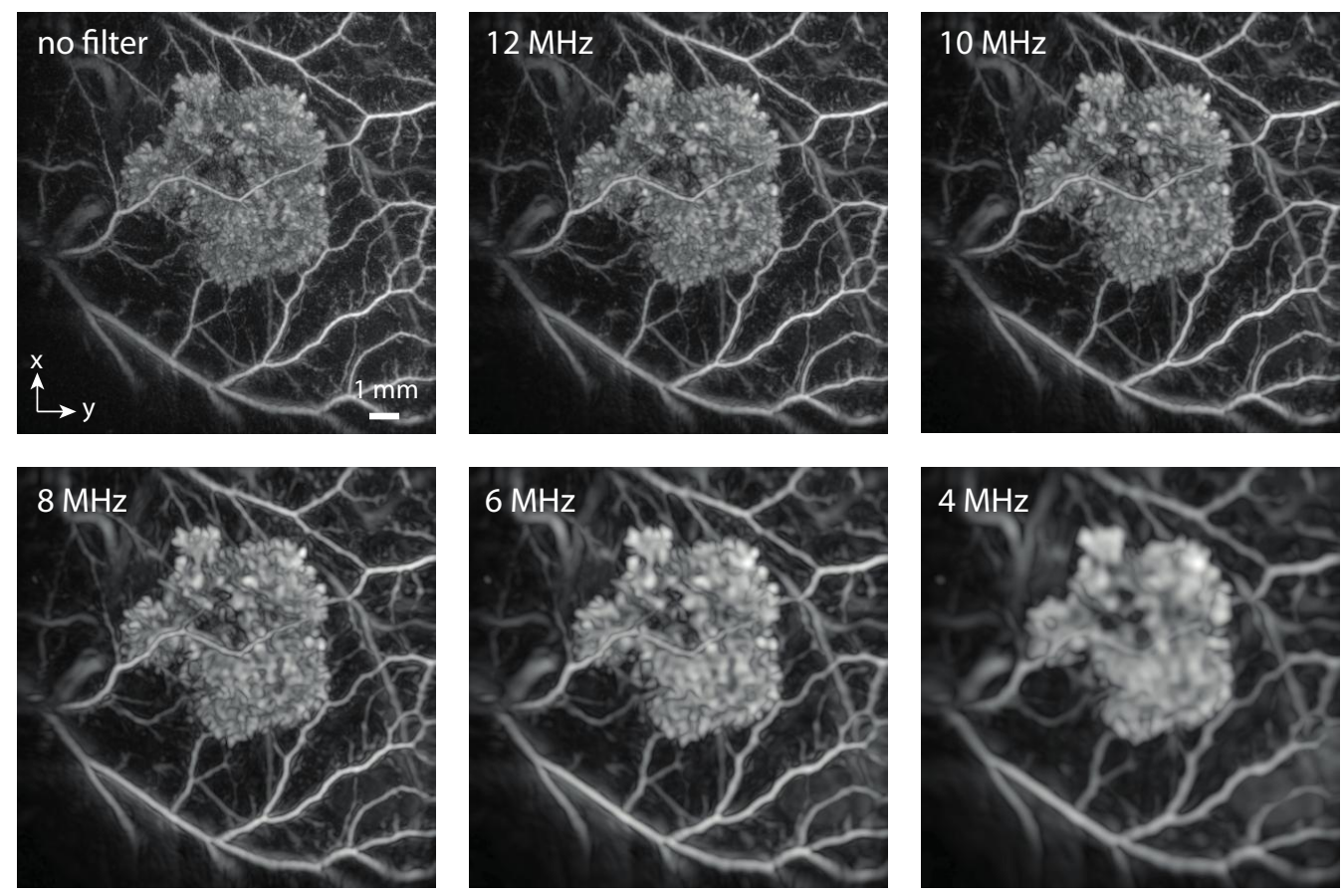

Figure 5. Reconstructed images using low-pass filtered data with filter cutoff frequencies from $12 \mathrm{MHz}$ to $4 \mathrm{MHz}$. 
A comparison of the images produced by these two methods is shown in Fig. 6. Because of the different assumptions inherent in the two algorithms, they produce visibly different images. First, the time reversal image has noticeably more detail, particularly in the tumour mass and smaller vasculature (e.g., the vessel denoted with the dashed arrows). This may be due to the way high-frequency information is mapped into the image in time reversal, and the inclusion of evanescent waves in the reconstruction. ${ }^{10}$ Second, the FFT-based image contains wrapping artefacts due to the assumed spatial periodicity of the data. This is clearly visible when the reconstruction is repeated after spatially zero-padding the time domain signals (right panels). For example, the vessel denoted with the solid arrow belongs at the top of the image outside the field of view (bottom right panel), but is mapped to the bottom of the FFT-based image without zero-padding (bottom left panel). The main advantage of the FFT-based algorithm is its speed. Consequently, it is used for the sound speed optimisation step discussed in Sec. 3.3, and real-time display. Time reversal is generally used for all other purposes.

\subsection{Image processing}

After reconstruction, very basic image processing is performed. First, a positivity condition is usually applied where negative acoustic pressures in the reconstructed image are thresholded to zero. ${ }^{27}$ Next, to improve the visibility of deeper lying vessels, a simple first-order correction for the variable light fluence in tissue is applied using a solution to the $1 \mathrm{D}$ diffusion equation

$$
\Phi(z)=\Phi_{0} \exp \left(-\mu_{\mathrm{eff}} z\right) .
$$

Here $\Phi(z)$ is the light fluence at depth $z$, and $\mu_{\text {eff }}$ is the effective attenuation coefficient, which can be in the range $50-250 \mathrm{~m}^{-1}$ depending on the type of tissue. Note, this assumes the optical illumination is a planar collimated beam at the top surface of the tissue, the fluence is diffuse everywhere, and the optical properties are constant throughout the tissue. ${ }^{7}$ These assumptions do not hold in general, thus this step does not quantitively correct for the spatial distribution of the fluence, but rather, qualitatively improves the visibility of deeper structures which in general will have received less light.

The effect of changing $\mu_{\text {eff }}$ on the lateral MIP of the tumour image is shown in Fig. 7. The right panels show 1D depth profiles summed across both lateral dimensions of the image, and give the total image intensity as a function of depth. If the image features were distributed evenly throughout the imaging volume, the peaks in the 1D profile would have approximately the same amplitude. However, because of optical attenuation, the image intensity rapidly decays with depth. As $\mu_{\mathrm{eff}}$ is increased, the visibility of deeper structures is improved and the image intensity becomes more uniform. However, this comes at the expense of increasing the noise level, particularly at greater depths in the image.

In addition to correction for optical attenuation, the image data is often log compressed to reduce the dynamic range of the image before display (analogous to the log compression performed in ultrasound imaging). The log compression is performed according to

$$
\bar{h}_{\text {compressed }}=\frac{\log _{10}\left(1+2^{l} \times \bar{h}\right)}{\log _{10}\left(1+2^{l}\right)},
$$

where $\bar{h}$ is the image data normalised between 0 and 1 , and $l$ is the compression level, which is typically set between 0 (low compression) and 4 (high compression). In $\mathrm{k}$-Wave, this is applied using the function logCompression. The nonlinear mapping given by Eq. (6) is plotted in Fig. 8(a), and the log compressed images using $l$ set to 1 and 4 are shown in Fig. 8(b). The compression makes it significantly easier to visualise the different structures in the image, particularly the small vasculature.

No other image processing (e.g., denoising) is routinely applied to the reconstructed images. In some cases, a manual segmentation and false colour might be used to highlight different regions of the image as shown in the left panel of Fig. 8(c). k-Wave also includes a vessel filtering function (vesselFilter), the output of which is shown in the middle panel of Fig. 8(c) ${ }^{28}$ However, this is less useful in the case of the tumour image, which appears almost cartoon like. Finally, in some cases a colour map is used for depth direction (en face) maximum intensity projections to illustrate the depth at which the maximum value is extracted. An example is shown in 
No Padding
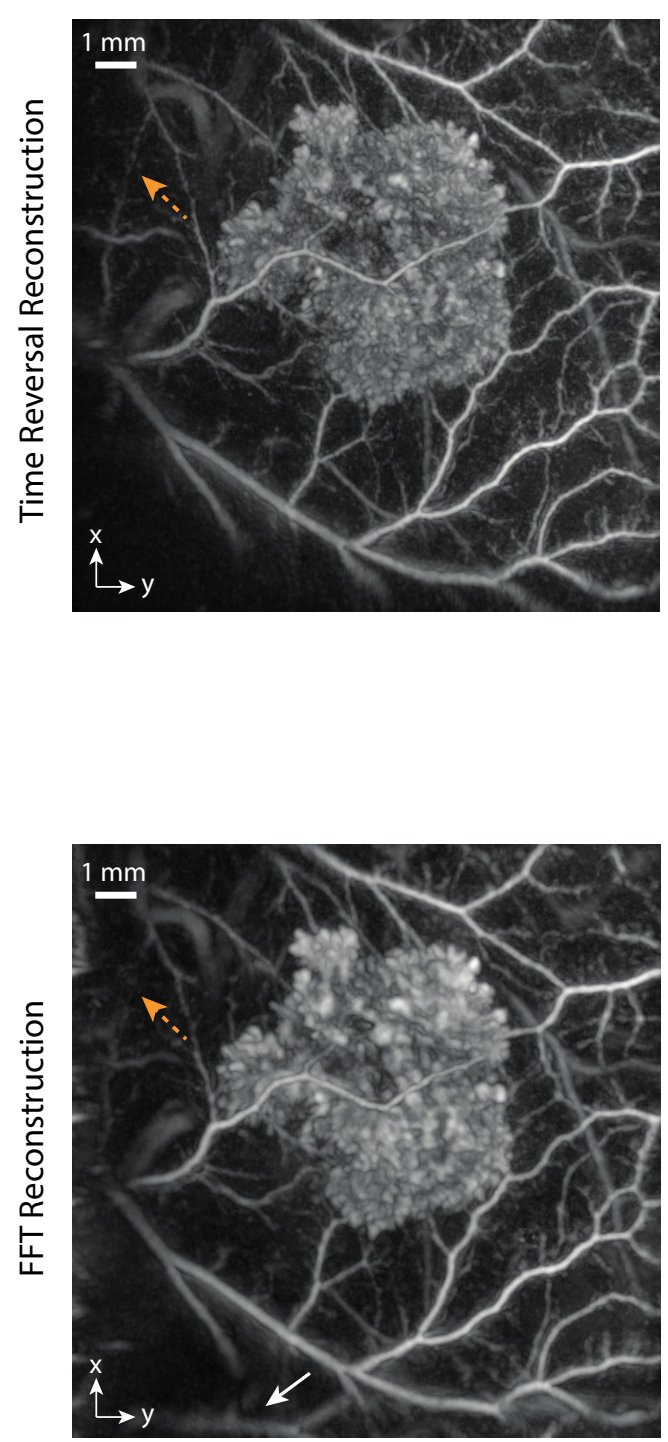

\section{Zero-Padded Before Reconstruction}
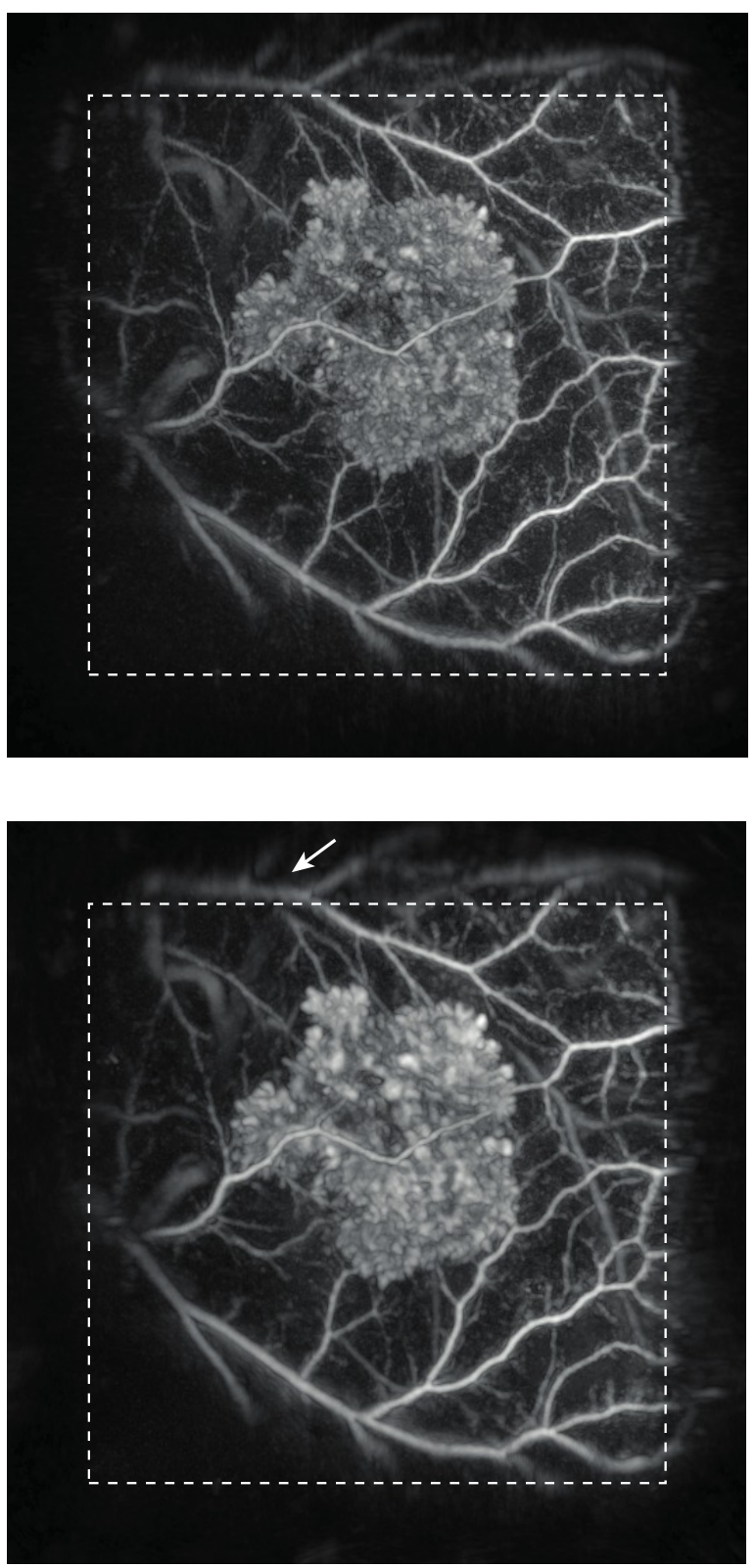

Figure 6. Comparison of images reconstructed using time reversal (top panels) and the FFT-based method (bottom panels) with and without zero padding the data. Time reversal gives a more detailed image (dashed arrows), and doesn't suffer from the wrapping artefacts present in the FFT-based reconstruction (solid arrows).

the right panel of Fig. 8(c). After processing, the 3D images are typically displayed as $2 \mathrm{D}$ maximum intensity projections. For depth-direction (en face) MIPs in particular, this can help reduce the visual perception of limited view artefacts. ${ }^{4}$ Note, if image resampling is needed for high-resolution display, this is performed using Fourier interpolation (interpftn in k-Wave) 

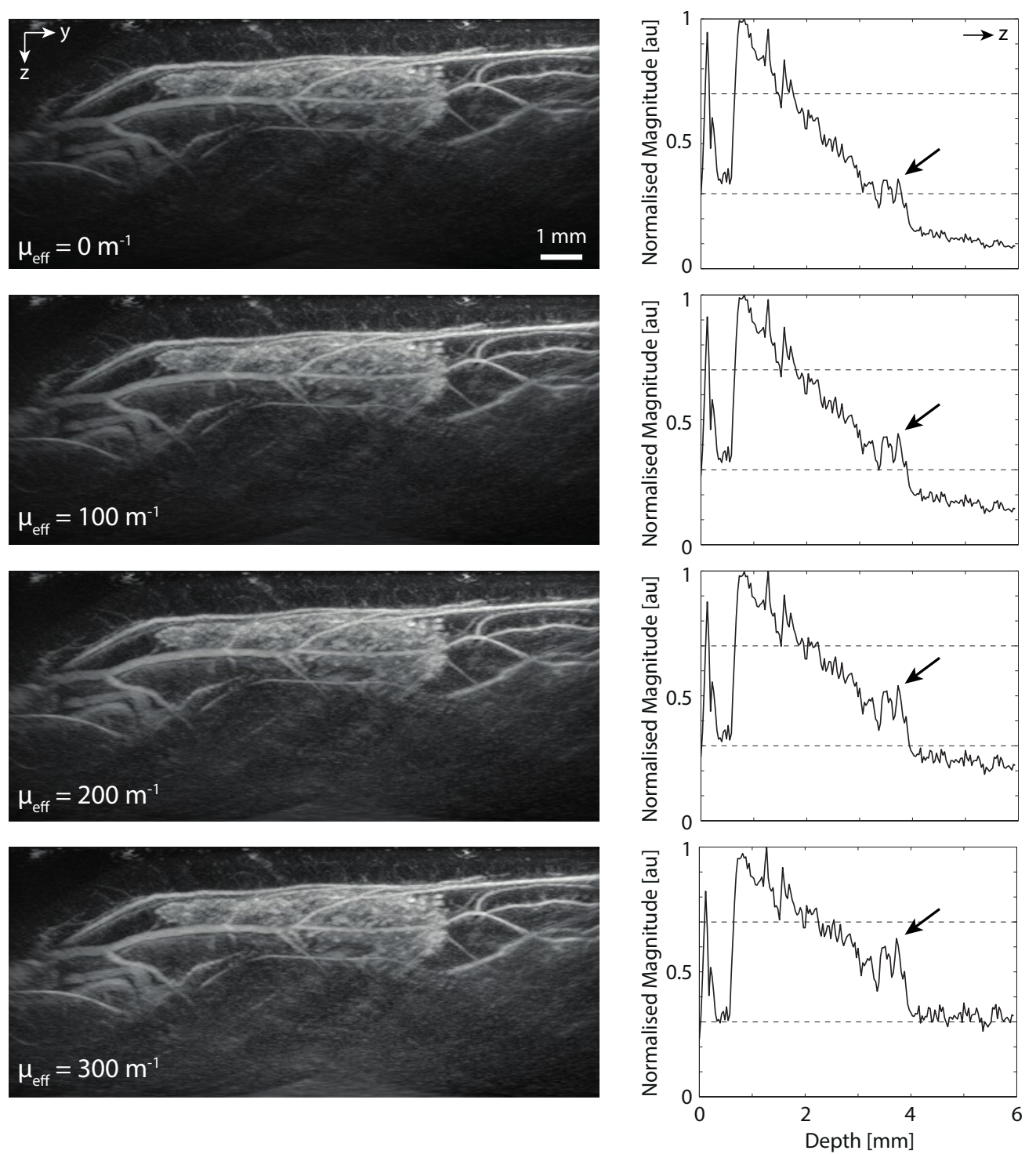

Figure 7. First order correction for optical attenuation using a solution to the 1D diffusion equation. The left panels show lateral maximum intensity projections through the reconstructed image using different values for the effective optical attenuation coefficient $\mu_{\text {eff }}$. The right panels show 1D depth profiles summed across both lateral dimensions of the image. As $\mu_{\text {eff }}$ is increased, the visibility of deeper vessels is improved (arrows), at the expense of increasing the noise level.

\section{SUMMARY}

The image reconstruction and processing methods used in photoacoustic tomography can have a significant impact on the quality, resolution, and clinical value of photoacoustic images. Consequently, in addition to optimising the light delivery and ultrasound detection systems, careful thought should also be given to the image reconstruction process. Techniques such as automatic sound speed selection and acoustic attenuation compensation are fast and easy to apply, and noticeably improve the reconstructed images. Using the latest hardware and software advances, three-dimensional time reversal image reconstruction can also be performed on relatively large datasets in under 30 seconds. All of the algorithms discussed are available in the open source $\mathrm{k}$-Wave toolbox, which makes it easy for others to apply them to their own datasets. 
(a)

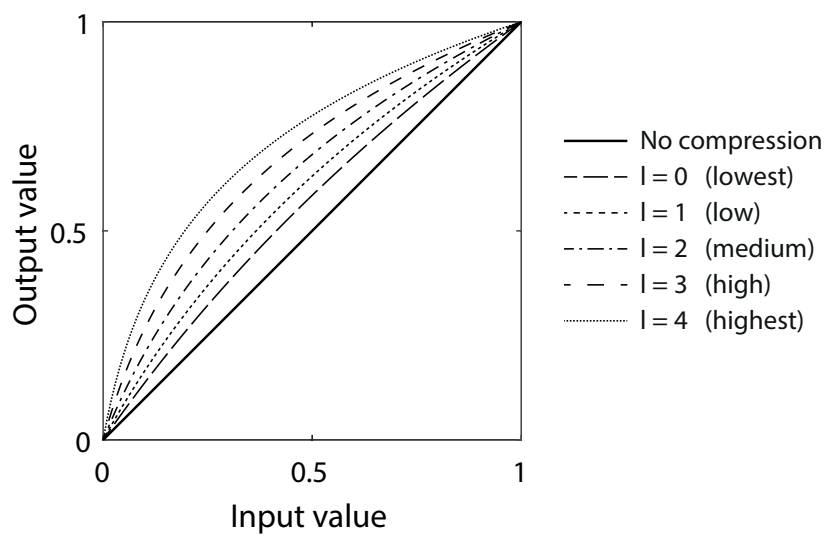

(b)
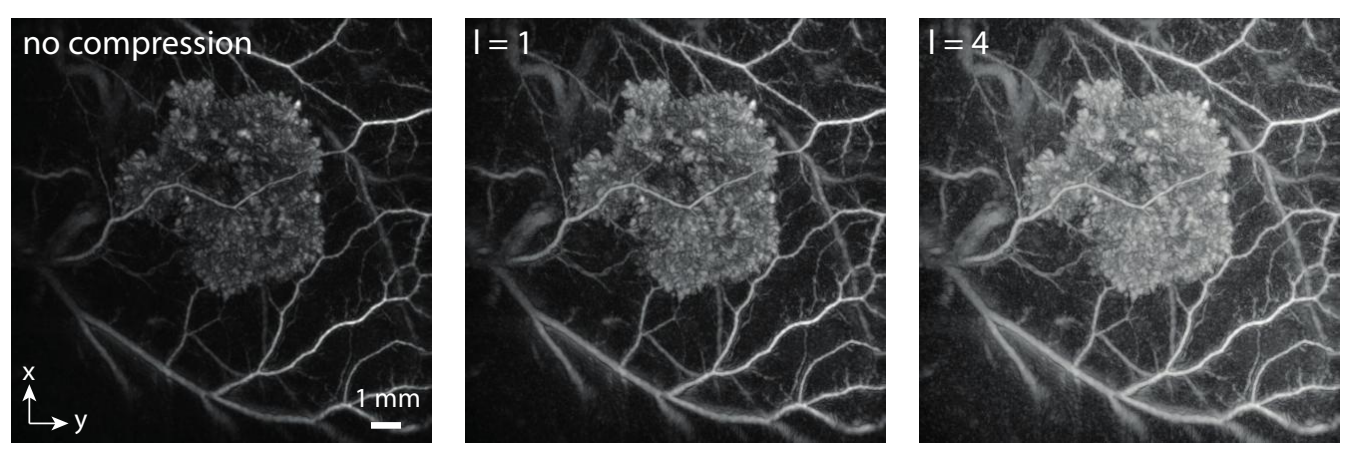

(c)
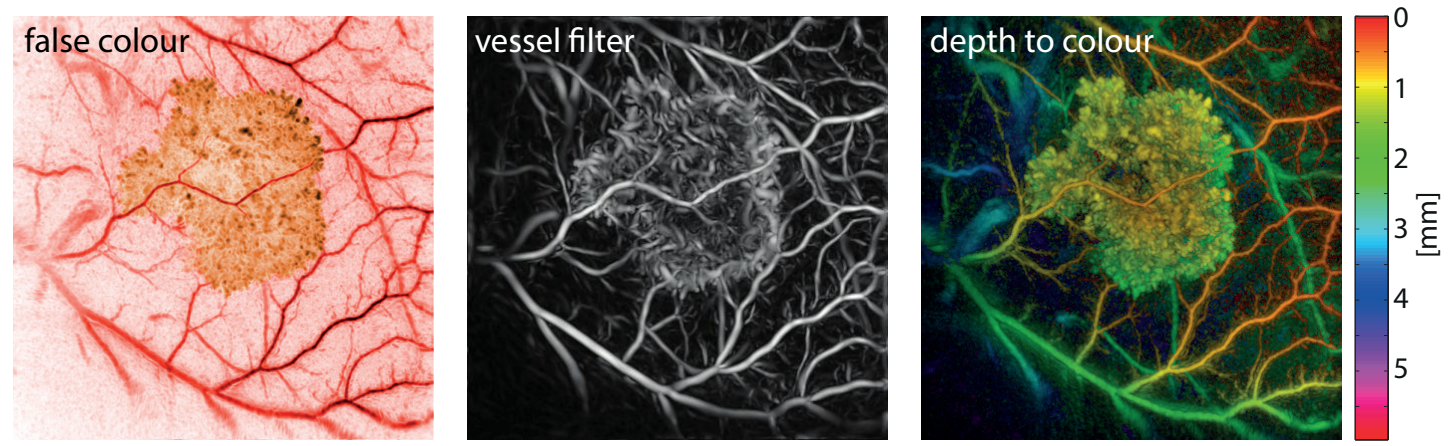

Figure 8. (a) Log compression curves for compression values from 0 to 4 calculated using Eq. (6). (b) Reconstructed photoacoustic images with no compression (left panel), log compression with $l=1$ (middle panel), and log compression with $l=4$ (right panel). (c) Other image processing techniques include false colour (left panel), vessel filtering (middle panel), and depth colour coded maximum intensity projections (right panel).

\section{ACKNOWLEDGMENTS}

The authors would like to thank Prof. Paul Beard, head of the Photoacoustic Imaging Group, for his contribution to the algorithms described in this paper, and valuable comments on the manuscript. This work was supported by the Engineering and Physical Sciences Research Council, UK (EP/L020262/1 and EP/M011119/1). The experimental data used in this study was provided by Amit Jathoul, Jan Laufer, Olumide Ogunlade, Bradley Treeby, Ben Cox, Edward Zhang, Peter Johnson, Arnold Pizzey, Brian Philip, Teresa Marafioti, Mark Lythgoe, R Barbara Pedley, Martin Pule, and Paul Beard. See Ref. 11 for details. Jiri Jaros is financed from the SoMoPro II Programme, co-financed by the European Union and the South-Moravian Region. This work reflects only the author's view and the European Union is not liable for any use that may be made of the information contained therein. 


\section{REFERENCES}

1. P. Beard, "Biomedical photoacoustic imaging," Interface Focus 1(4), pp. 602-631, 2011.

2. M. Lakshman and A. Needles, "Screening and quantification of the tumor microenvironment with microultrasound and photoacoustic imaging," Nat. Meth. 12(4), pp. iii-v, 2015.

3. P. Kuchment and L. Kunyansky, "Mathematics of Photoacoustic and Thermoacoustic Tomography," in Handbook of Mathematical Methods in Imaging, ch. 19, pp. 817-865, Springer, 2011.

4. Y. Xu, L. V. Wang, G. Ambartsoumian, and P. Kuchment, "Reconstructions in limited-view thermoacoustic tomography," Med. Phys. 31(4), pp. 724-733, 2004.

5. B. T. Cox and B. E. Treeby, "Effect of sensor directionality on photoacoustic imaging: A study using the k-Wave toolbox," in Proc. of SPIE, 7564, pp. 6-11, 2010.

6. N. A. Rejesh, H. Pullagurla, and M. Pramanik, "Deconvolution-based deblurring of reconstructed images in photoacoustic/thermoacoustic tomography," J. Opt. Soc. Am. A 30(10), pp. 1994-2001, 2013.

7. B. Cox, J. G. Laufer, S. R. Arridge, and P. C. Beard, "Quantitative spectroscopic photoacoustic imaging: A review," J. Biomed. Opt. 17(6), p. 061202, 2012.

8. C. Yoon, J. Kang, S. Han, Y. Yoo, T.-K. Song, and J. H. Chang, "Enhancement of photoacoustic image quality by sound speed correction: Ex vivo evaluation," Opt. Express 20(3), pp. 3082-3090, 2012.

9. D. Van de Sompel, L. S. Sasportas, A. Dragulescu-Andrasi, S. Bohndiek, and S. S. Gambhir, "Improving image quality by accounting for changes in water temperature during a photoacoustic tomography scan," PloS one $\mathbf{7}(10)$, p. e45337, 2012.

10. B. E. Treeby and B. T. Cox, "k-Wave: MATLAB toolbox for the simulation and reconstruction of photoacoustic wave fields," J. Biomed. Opt. 15(2), p. 021314, 2010.

11. A. P. Jathoul, J. Laufer, O. Ogunlade, B. Treeby, B. Cox, E. Zhang, P. Johnson, A. R. Pizzey, B. Philip, T. Marafioti, M. F. Lythgoe, R. B. Pedley, M. A. Pule, and P. Beard, "Deep in vivo photoacoustic imaging of mammalian tissues using a tyrosinase-based genetic reporter," Nat. Photon. 9, pp. 239-246, 2015.

12. E. Zhang, J. Laufer, and P. Beard, "Backward-mode multiwavelength photoacoustic scanner using a planar Fabry-Perot polymer film ultrasound sensor for high-resolution three-dimensional imaging of biological tissues," Appl. Optics 47(4), pp. 561-577, 2008.

13. J. Laufer, P. Johnson, E. Zhang, B. Treeby, B. Cox, B. Pedley, and P. Beard, "In vivo preclinical photoacoustic imaging of tumor vasculature development and therapy," J. Biomed. Opt. 17(5), p. 056016, 2012.

14. J. Laufer, F. Norris, J. Cleary, E. Zhang, B. Treeby, B. Cox, P. Johnson, P. Scambler, M. Lythgoe, and P. Beard, "In vivo photoacoustic imaging of mouse embryos," J. Biomed. Opt. 17(6), p. 061220, 2012.

15. S. P. Johnson, O. Ogunlade, E. Zhang, J. Laufer, V. Rajkumar, R. B. Pedley, and P. Beard, "Photoacoustic tomography of vascular therapy in a preclinical mouse model of colorectal carcinoma," in Proc. of SPIE, 8943, p. 89431R, 2014.

16. P. Burgholzer, H. Grün, M. Haltmeier, R. Nuster, and G. Paltauf, "Compensation of acoustic attenuation for high resolution photoacoustic imaging with line detectors," in Proc. of SPIE, 6437, p. 643724, 2007.

17. B. E. Treeby, E. Z. Zhang, and B. T. Cox, "Photoacoustic tomography in absorbing acoustic media using time reversal," Inverse Probl. 26(11), p. 115003, 2010.

18. B. E. Treeby, "Acoustic attenuation compensation in photoacoustic tomography using time-variant filtering," J. Biomed. Opt. 18(3), p. 036008, 2013.

19. T. L. Szabo, Diagnostic Ultrasound Imaging, Elsevier Academic Press, London, 2004.

20. B. E. Treeby, J. G. Laufer, E. Z. Zhang, F. C. Norris, M. F. Lythgoe, P. C. Beard, and B. T. Cox, "Acoustic attenuation compensation in photoacoustic tomography: Application to high-resolution 3D imaging of vascular networks in mice," in Proc. of SPIE, 7899, p. 78992Y, 2011.

21. B. E. Treeby, T. K. Varslot, E. Z. Zhang, J. G. Laufer, and P. C. Beard, "Automatic sound speed selection in photoacoustic image reconstruction using an autofocus approach," J. Biomed. Opt. 16(9), p. 090501, 2011.

22. B. E. Treeby, J. Jaros, A. P. Rendell, and B. T. Cox, "Modeling nonlinear ultrasound propagation in heterogeneous media with power law absorption using a k-space pseudospectral method," J. Acoust. Soc. Am. 131(6), pp. 4324-4336, 2012. 
23. S. J. Norton and M. Linzer, "Ultrasonic reflectivity imaging in three dimensions: Exact inverse scattering solutions for plane, cylindrical, and spherical apertures," IEEE T. Biomed. Eng. 28(2), pp. 202-220, 1981.

24. K. P. Koestli, M. Frenz, H. Bebie, H. P. Weber, K. P. Köstli, M. Frenz, H. Bebie, and H. P. Weber, "Temporal backward projection of optoacoustic pressure transients using Fourier transform methods," Phys. Med. Biol. 46(7), pp. 1863-1872, 2001.

25. D. Finch, S. K. Patch, and Rakesh, "Determining a function from its mean values over a family of spheres," SIAM J. Math. Anal. 35(5), pp. 1213-1240, 2004.

26. Y. Xu and L. Wang, "Time reversal and its application to tomography with diffracting sources," Phys. Rev. Lett. 92(3), pp. 3-6, 2004.

27. G. Paltauf, J. A. Viator, S. A. Prahl, and S. L. Jacques, "Iterative reconstruction algorithm for optoacoustic imaging," J. Acoust. Soc. Am. 112(4), p. 1536, 2002.

28. T. Oruganti, J. Laufer, and B. E. Treeby, "Vessel filtering of photoacoustic images," in Proc. of SPIE, 8581, p. 85811W, 2013. 